\title{
Prolonged constipation and diarrhea in childhood and disordered eating in adolescence
}

\author{
Camilla A. Wiklund ${ }^{\mathrm{a}}$, Ralf Kuja-Halkola ${ }^{\mathrm{a}}$, Laura M. Thornton ${ }^{\mathrm{b}}$, Christopher Hübel ${ }^{\mathrm{a}, \mathrm{c}}$, Virpi Leppäa \\ Cynthia M. Bulik ${ }^{\mathrm{a}, \mathrm{b}, \mathrm{d}, *}$ \\ ${ }^{a}$ Department of Medical Epidemiology and Biostatistics, Karolinska Institutet, Stockholm, Sweden \\ ${ }^{\mathrm{b}}$ Department of Psychiatry, University of North Carolina at Chapel Hill, Chapel Hill, NC, USA \\ ' Social, Genetic \& Developmental Psychiatry Centre, Institute of Psychiatry, Psychology \& Neuroscience, King's College London, London, UK \\ ${ }^{\mathrm{d}}$ Department of Nutrition, University of North Carolina at Chapel Hill, Chapel Hill, NC, USA
}

\section{A R T I C L E I N F O}

Keywords:

Adolescents

Children

Eating disorders

Gastrointestinal problems

\begin{abstract}
A B S T R A C T
Objectives: Gastrointestinal problems are common in all eating disorders; however, the extent to which these problems predate the onset of eating disorders is not clear. We explored longitudinal associations between childhood gastrointestinal problems and adolescent disordered eating, and assessed whether observed associations are potentially causal or due to familial confounding factors.

Methods: Data from a population-based Swedish twin sample were used to investigate associations between parent- and self-reported protracted constipation and diarrhea in childhood and adolescence, and later disordered eating, measured by the Eating Disorders Inventory-2 (EDI). Linear regression models were used to investigate the associations. Possible familial confounding was explored by using a within-twin pair analysis. Results: We found that those who reported a history of constipation at age 15 scored 5.55 and 5.04 points higher, respectively, on the EDI total score at age 15 and 18, compared with those without constipation. Those reporting a history of diarrhea at age 15 scored 5.15 points higher, and the group reporting both problems scored 9.52 points higher on the EDI total score at age 15 than those reporting no problems. We observed that the association between constipation and disordered eating was attenuated in the within-twin pair analysis, but remained positive.

Conclusions: Gastrointestinal problems in childhood and adolescence are significantly associated with disordered eating. Associations were partly due to familial confounding, but might also be consistent with a causal interpretation. Clinicians should be aware of the increased risk of disordered eating when following children and adolescents who present with gastrointestinal problems.
\end{abstract}

\section{Introduction}

Gastrointestinal (GI) problems such as bloating, nausea, and epigastric discomfort commonly occur in individuals with eating disorders including anorexia nervosa, bulimia nervosa, and binge-eating disorder [1-7]. In fact, $67-83 \%$ of patients with anorexia nervosa and $63 \%$ of patients with bulimia nervosa report constipation, and $41-52 \%$ of patients with eating disorders report irritable bowel syndrome (IBS) [4]. However, the extent to which these problems predate the onset of eating disorders has not been thoroughly researched.

In the general population, GI problems in childhood are relatively common, with prevalence estimates ranging from 8 to $25 \%$ varying by type of sample, nature of problem, and age $[8,9]$. The long-term implications of childhood GI problems are less clear, although pediatric recurrent abdominal pain is a known precursor of functional GI disorders in adulthood, especially IBS [10,11]. In addition, individuals with recurrent abdominal pain have also been shown to develop both chronic abdominal pain, as well as psychiatric disorders later in life $[12,13]$. Further, children with recurrent abdominal pain are reported to have more concurrent psychiatric illnesses, including depressive and anxiety disorders [14].

Täljemark et al. reported that, in children, psychiatric and GI problems often coexist with restrictive eating accompanied by fear of weight gain [15]. In addition, results from a population-wide Danish

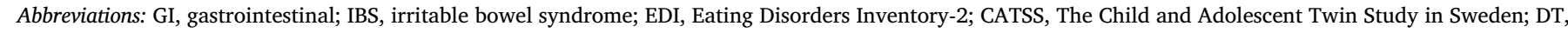
drive for thinness; B, bulimia; BD, body dissatisfaction; CI, confidence interval; DZ, dizygotic; MZ, monozygotic

* Corresponding author at: Department of Medical Epidemiology and Biostatistics, Karolinska Institutet, Nobels väg $12 a$, 17177 Stockholm, Sweden.

E-mail address: cynthia bulik@med.unc.edu (C.M. Bulik). 
study of children and adolescents reported an increased risk of eating disorders in individuals with autoimmune diseases with GI involvement, such as Crohn's disease and celiac disease [16].

In this study, we evaluated the longitudinal associations between two common GI problems (i.e., prolonged constipation and prolonged diarrhea) during childhood and eating disorders symptoms in adolescence and assessed whether these associations are potentially causal or more likely to reflect familial confounding using a large longitudinal, population-based twin sample. Familial confounding is defined as factors that are shared within a family or, for this study, within twin pairs (e.g., genetic factors, socioeconomic factors, parental education, inutero effects), that may influence the association of interest. We use a within-twin pair analysis where we condition the analysis on the pairs including differently exposed twins. We can thereby compare the association in the full sample to the potential association within twin pairs. We hypothesized that GI problems in childhood would be associated with elevated risk of disordered eating in adolescence as measured by three scales of Eating Disorders Inventory-2 (EDI) [17].

\section{Method}

\subsection{Population}

The Child and Adolescent Twin Study in Sweden (CATSS) is an ongoing, population-based, longitudinal study following twins born in Sweden since 1992 [18]. CATSS enrolment has been ongoing since July 2004. Every year, parents of all twins who are turning 9 years old in Sweden are invited to participate in a telephone interview regarding the children's mental and physical health (i.e., CATSS began with twins born in July 1995 and onwards). During the first three years of CATSS ascertainment (2004-2006), parents of 12-year-old twins (i.e., twins born in July 1992 to June 1995) were also invited to participate in parallel to the 9 -year-olds. In subsequent years, only twins turning 9 were ascertained. In total, $23 \%$ of the sample at this timepoint had their telephone interviews conducted at age 12 and the remainder at age 9 . Both the twins and their parents were then asked to participate in follow-up assessments using online questionnaires at age 15 and 18. The response rate in CATSS is approximately $75 \%$ at age $9 / 12$ and $60 \%$ at ages 15 and 18. Parents provided informed consent before the interview, and twins provided informed consent before participating in the follow-up. CATSS has been approved by the Regional Ethical Review Board in Stockholm, Sweden.

Zygosity was determined using a panel of 48 single nucleotide polymorphisms when DNA from saliva was available [19]. When DNA was not available, an algorithm based on five questions on twin similarity derived from pairs with known zygosity was used [20]. The algorithm classifies $>95 \%$ of twins correctly compared to DNA testing [19].

\subsection{Measures}

\subsubsection{GI problems}

The CATSS cohort includes approximately 28,000 twins and assesses a broad array of child health, mental health, and behavioral and developmental areas. Given clinical observations and clinical histories of patients with eating disorders, we were particularly interested in the relationship between GI-related issues and disordered eating behavior. We identified two questions that were administered on more than one occasion in CATSS that capture GI problems in childhood: "Does (s)he have or has (s)he ever had problems with prolonged constipation growing up?" and "Does (s)he have or has (s)he ever had problems with prolonged diarrhea growing up?". The questions refer to the entire childhood period. Parents reported these symptoms during the telephone interview at age 9 or 12. Response options are "yes" or "no". Options "Don't know" and "Don't want to answer" were coded as missing values. At age 15, the twins themselves answered the same questions about constipation and diarrhea. We also created a variable encompassing both problems, C/D: those who self-reported having both constipation and diarrhea at age 15 were scored positively, all others were scored negatively. Individuals were considered "exposed" for a specific GI problem if they scored positively for that problem. Those who scored negatively were considered to be "unexposed".

\subsubsection{Disordered eating}

At ages 15 and 18, twins reported disordered eating using the three subscales that measure eating-related attitudes and behaviors from the EDI, a self-report scale. We evaluated the EDI total score, defined as the sum of the three sub-scales assessed-Drive for thinness (DT), Bulimia (B), and Body dissatisfaction (BD)—and each sub-scale separately. These three sub-scales are designed to measure core eating disorder symptoms and have been validated in both clinical and non-clinical settings in Scandinavia, showing high internal reliability and the ability to distinguish between a patient- and a healthy population [21-23]. Each sub-scale includes several items answered on a 6-point Likert scale ranging from "Always" to "Never". We use the full 6-point scale in order to capture the full variation of disordered eating in the population. The total score and the sub-scale scores were analyzed as continuous variables. In the CATSS data collection, one item from the BD subscale is missing (I like the shape of my buttocks); however, the reverse item ("I think my buttocks are too large") is included. Thus, the highest possible scores were $\mathrm{DT}=42, \mathrm{~B}=42$, and $\mathrm{BD}=48$ with higher scores indicating more disordered eating. This may result in lower EDI total and BD sub-scale scores but should not affect study results as all participants received the same assessment version. However, it should be considered when comparing this study to other studies using the same scales.

\subsection{Statistical analysis}

The prevalence for each GI measure at each age, for the total sample, and by sex was calculated. We further calculated the mean EDI total score at ages 15 and 18 for exposed and unexposed individuals in the total sample and by sex.

To investigate the influence of prior GI problems on disordered eating, we applied linear regression models, where GI problems were the exposures and disordered eating scores were the outcomes in all analyses. Generalized estimating equations were used to correct the standard errors for familial clustering of twins using a cluster-robust sandwich estimator, and there were no assumptions of a normal distribution [24]. Specifically, we tested whether the EDI total scores at ages 15 and 18 were associated with GI problems at ages 9/12 and 15 (crude models). We then controlled for sex in the analyses (adjusted models) to determine if sex moderated any association. Regression coefficients and 95\% confidence intervals (CIs) were calculated based on robust standard errors. For hypothesis testing, we focus on the primary analyses of the study, namely the adjusted models among constipation, diarrhea, and C/D on total EDI score, i.e., 10 tests (second column in Tables 2 and 3). We therefore set the statistical significance threshold at $p<.005$ to adjust for multiple comparisons.

To investigate whether genetic/familial confounding accounted for associations between GI problems and disordered eating, we applied conditional linear regression models (within-twin pair analysis). Specifically, we adjusted for unmeasured familial confounding factors, meaning both genetic and environmental factors that are shared between the individuals in the twin pair, and that could account for a potential association between GI problems and disordered eating. Examples of these factors could be shared genetic background, or environmental exposures to which twins are equally exposed. When using within-twin pair analysis, we compared dizygotic (DZ) and monozygotic (MZ) twin pairs, respectively, who were discordant for GI problems, thereby controlling for these familial confounding factors that are constant within the twin pair.

If GI problems and disordered eating are associated because GI 
problems play a causal role in the difference in mean EDI score between the exposed and unexposed group, we would expect the association to be similar in the total sample and within the twin pairs, suggesting that genetic similarities between the twins would be of no significance and would therefore not influence the association. Conversely, if the association between exposure and outcome was greater at the total sample level than the association in DZ pairs, and further attenuated in MZ pairs, there would be reason to assume that the association would be better explained by genetic and/or familial confounding factors [25]. Further, we would expect no association in MZ pairs if the association were entirely due to genetic and/or familial confounding factors.

The associations between the different EDI sub-scales and self-rated GI problems at age 15 were also explored using these methods.

Because of the difficulty in de termining the te mporality of a potential association, we performed a sensitivity analysis between GI problems at age 15 and total EDI score at age 18 adjusting for total EDI score at age 15 .

All analyses were done using $\mathrm{R}$ version 3.2.2 [26] using the package drgee [27].

\section{Results}

\subsection{Prevalence of GI problems and mean EDI scores}

The prevalence of constipation and diarrhea at age 9/12 and age 15 in the full sample and by sex are presented in Table 1. Constipation was more common than diarrhea at both ages, constipation was reported in $8.5 \%$ and $7.3 \%$ at age $9 / 12$ and age 15 , respectively, and diarrhea reported in $3.4 \%$ and $3.0 \%$ at the same ages. Constipation was also more prevalent in girls reported in $10.0 \%$ at age $9 / 12$ and $8.9 \%$ at age 15 compared to boys who reported constipation in $7.0 \%$ and $5.2 \%$, at the two ages, (all $p$-values $<.001$ ). In contrast, more boys $(4.1 \%)$ than girls $(3.2 \%)$ reported diarrhea age $9 / 12$ (p-value $<.001$ ). Reporting C/D conditions was slightly more common in girls than boys $(1.1 \%$ vs. $0.7 \%)$ (p-value $=.020$ ).

Table 1 also presents the mean EDI total score for those reporting constipation, diarrhea, or $\mathrm{C} / \mathrm{D}$, and for unexposed twins. Estimates are presented for the full sample and for girls and boys separately, at ages 15 and 18.

\subsection{Developmental associations between GI problems and eating disorder symptoms}

No association was found between constipation or diarrhea at age 9/12 and EDI score at age 15 or 18 . We therefore did not explore the combined GI conditions (C/D).

Linear regression (Table 2) showed an association between constipation and diarrhea at age 15 and EDI total at age 15 with estimated adjusted regression coefficients of 5.55 (95\% CI: 3.77; 7.33) and 5.15 (95\% CI: 2.74; 7.55), respectively. Thus, those who reported constipation or diarrhea scored about 5 points higher on the EDI total scale than the unexposed group. Reporting both GI conditions (C/D) at age 15 was also associated with a significantly higher EDI total score (regression coefficient: 9.52, 95\% CI: 4.27; 14.75) compared to the unexposed group. Constipation at age 15 was significantly associated with EDI total score at age 18 (regression coefficient: 5.04, 95\% CI: 1.79; 8.10) (Table 3). Associations of diarrhea and C/D conditions at age 15 with EDI total score at age 18 were not statistically significant.

\subsection{Contribution of familial confounding to the association}

Within-twin pair analysis of constipation and EDI total score, both measured at age 15 (Table 2), showed lower, but not null, estimates for both DZ and MZ pairs (DZ regression coefficient: 2.12, 95\% CI: -0.78 ; 5.03 and MZ regression coefficient: 4.53, 95\% CI: 0.68; 8.39) compared with the crude and adjusted estimates; however, these were not statistically significant at $p<.005$ level. This can be interpreted as constipation having a strong association with EDI total score at age 15, and that the association is partly explained by familial/genetic factors. However, constipation at age 15 and EDI total score at age 18 (Table 3) were not significantly associated in the within-twin pair analysis. The conditioned analysis included a smaller number of twins (DZ: $n=185$, MZ: $n=65$ ).

No statistically significant association was found between self-reported diarrhea and EDI total score, both measured at age 15 (Table 2), in the within-twin pair analysis. The interpretation is therefore that the previously observed association can largely be explained by familial (genetic and/or environmental) confounding. The same analysis for diarrhea at age 15 and EDI total score at age 18 showed no significant associations; however, the confidence intervals were very wide.

Associations between C/D conditions and EDI total score at age 15 were not statistically significant. Few individuals reported C/D conditions, which influenced power to detect potential associations (DZ: $n=71$, MZ: $n=25$ ).

\subsection{Associations between GI problems and the EDI sub-scales}

We further examined the association between constipation and diarrhea reported at age 15 and the three EDI sub-scales at ages 15 and 18 (Table 4). The results followed the same patterns as the main results,

Table 1

Prevalence of GI problems [constipation, diarrhea, and constipation and diarrhea (C/D)] at ages 9/12 and 15 in the total population and by sex, in CATSS. Mean (sd) EDI total score at ages 15 and 18 in the total population and by sex for the different GI problems at ages 9/12 and 15 .

\begin{tabular}{|c|c|c|c|c|c|c|c|c|c|c|}
\hline & \multicolumn{2}{|c|}{$\begin{array}{l}\text { Constipation age } 9 / 12 \\
\text { (parent) }\end{array}$} & \multicolumn{2}{|c|}{$\begin{array}{l}\text { Diarrhea age } 9 / 12 \\
\text { (parent) }\end{array}$} & \multicolumn{2}{|c|}{$\begin{array}{l}\text { Constipation age } 15 \\
\text { (self) }\end{array}$} & \multicolumn{2}{|c|}{$\begin{array}{l}\text { Diarrhea age } 15 \\
\quad \text { (self) }\end{array}$} & \multicolumn{2}{|c|}{$\begin{array}{l}\text { C/D age } 15 \\
\text { (self) }\end{array}$} \\
\hline & $\begin{array}{l}\text { Yes } \\
\mathrm{N}(\%)\end{array}$ & $\begin{array}{l}\text { No } \\
\text { N (\%) }\end{array}$ & $\begin{array}{l}\text { Yes } \\
\mathrm{N}(\%)\end{array}$ & $\begin{array}{l}\text { No } \\
\text { N (\%) }\end{array}$ & $\begin{array}{l}\text { Yes } \\
\mathrm{N}(\%)\end{array}$ & $\begin{array}{c}\text { No } \\
\text { N (\%) }\end{array}$ & $\begin{array}{l}\text { Yes } \\
\mathrm{N}(\%)\end{array}$ & $\begin{array}{l}\text { No } \\
\text { N (\%) }\end{array}$ & $\begin{array}{l}\text { Yes } \\
\mathrm{N}(\%)\end{array}$ & $\begin{array}{c}\text { No } \\
\text { N (\%) }\end{array}$ \\
\hline All & $2411(8.5)$ & 26,015 (91.5) & $961(3.4)$ & $27,476(96.6)$ & $608(7.3)$ & $8330(92.7)$ & $263(3.0)$ & $8713(97.0)$ & $83(0.9)$ & $8832(99.1)$ \\
\hline Girls & $1394(10.0)$ & $12,555(90.0)$ & $366(2.3)$ & $13,592(97.4)$ & $337(8.9)$ & $3781(91.1)$ & $107(2.7)$ & $4032(97.3)$ & $44(1.1)$ & 4086 (98.9) \\
\hline Boys & $1017(7.0)$ & $13,460(93.0)$ & $595(4.1)$ & $13,884(95.9)$ & $172(5.2)$ & $3331(94.8)$ & $110(3.2)$ & $3411(96.8)$ & $25(0.7)$ & 3479 (99.3) \\
\hline EDI 15 & \multicolumn{2}{|c|}{ Mean (sd) } & \multicolumn{2}{|c|}{ Mean (sd) } & \multicolumn{2}{|c|}{ Mean (sd) } & \multicolumn{2}{|c|}{ Mean (sd) } & \multicolumn{2}{|c|}{ Mean (sd) } \\
\hline Boys & $37.7(11.5)$ & $38.5(11.4)$ & $38.4(11.4)$ & $40.4(12.7)$ & $41.3(15.5)$ & $38.1(11.0)$ & $43.0(15.6)$ & $38.1(11.0)$ & $49.9(24.3)$ & $38.4(11.2)$ \\
\hline EDI 18 & \multicolumn{2}{|c|}{ Mean (sd) } & \multicolumn{2}{|c|}{ Mean (sd) } & \multicolumn{2}{|c|}{ Mean (sd) } & \multicolumn{2}{|c|}{ Mean (sd) } & \multicolumn{2}{|c|}{ Mean (sd) } \\
\hline All & $55.4(20.6)$ & $51.3(19.0)$ & $49.5(20.3)$ & $51.6(19.0)$ & $58.8(20.5)$ & $51.8(19.2)$ & $54.4(18.4)$ & $52.1(19.3)$ & $62.1(19.7)$ & $51.9(19.2)$ \\
\hline Girls & $60.7(20.8)$ & $58.6(19.6)$ & $59.2(22.9)$ & $58.8(19.6)$ & $63.8(21.1)$ & $58.4(19.9)$ & $59.3(20.7)$ & $58.7(19.9)$ & $65.9(18.8)$ & $58.7(19.7)$ \\
\hline Boys & $43.5(14.5)$ & $41.7(12.8)$ & $42.3(14.6)$ & $41.8(12.9)$ & $46.1(14.9)$ & $42.1(13.2)$ & $44.7(10.3)$ & $42.2(13.4)$ & $43.2(12.8)$ & $41.8(12.9)$ \\
\hline
\end{tabular}

CATSS $=$ the Child and Adolescent Twin Study in Sweden, self $=$ self-report, parent $=$ parent-report, sd $=$ standard deviation, EDI $=$ Eating Disorders Inventory-2, $\mathrm{C} / \mathrm{D}=$ reporting both constipation and diarrhea, $\mathrm{GI}=$ gastrointestinal. 
Table 2

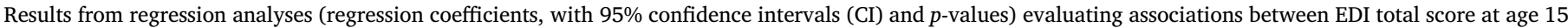

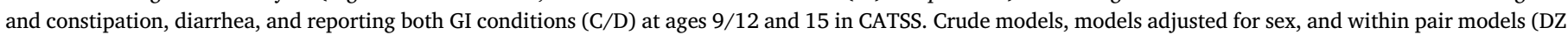
and MZ twin) are presented.

\begin{tabular}{|c|c|c|c|c|}
\hline & Crude $(95 \% \mathrm{CI})$ & $\begin{array}{l}\text { Adjusted }^{\mathrm{a}} \\
(95 \% \mathrm{CI})\end{array}$ & $\begin{array}{l}\text { Within DZ pairs }^{\mathrm{a}} \\
(95 \% \mathrm{CI}) \\
{\left[\mathrm{N}=\text { exposure discordant twins }{ }^{\mathrm{b}}\right]}\end{array}$ & $\begin{array}{l}\text { Within MZ pairs } \\
(95 \% \mathrm{CI}) \\
{\left[\mathrm{N}=\text { exposure discordant twins }{ }^{\mathrm{b}}\right]}\end{array}$ \\
\hline & \multicolumn{4}{|l|}{ EDI total score age 15} \\
\hline Constipation age $9 / 12$ (parent) & $\begin{array}{l}1.37(-0.31 ; 3.05) \\
(p=0.111)\end{array}$ & $\begin{array}{l}-0.05(-1.57 ; 1.47) \\
(\mathrm{p}=0.950)\end{array}$ & $\begin{array}{l}-2.03(-4.23 ; 0.16) \\
{[\mathrm{N}=683]} \\
(\mathrm{p}=0.069)\end{array}$ & $\begin{array}{l}1.11(-2.59 ; 4.81) \\
{[N=176]} \\
(p=0.556)\end{array}$ \\
\hline Diarrhea age 9/12 (parent) & $\begin{array}{l}-0.27(-2.88 ; 2.33) \\
(\mathrm{p}=0.837)\end{array}$ & $\begin{array}{l}1.55(-0.84 ; 3.94) \\
(p=0.204)\end{array}$ & $\begin{array}{l}1.26(-2.60 ; 5.12) \\
{[\mathrm{N}=270]} \\
(\mathrm{p}=0.523)\end{array}$ & $\begin{array}{l}-5.00(-9.64 ;-0.36) \\
{[N=73]} \\
(p=0.034)\end{array}$ \\
\hline Constipation age 15 (self) & $\begin{array}{l}7.39(5.47 ; 9.31) \\
(p<0.001)^{*}\end{array}$ & $\begin{array}{l}5.55(3.77 ; 7.33) \\
(p<0.001)^{*}\end{array}$ & $\begin{array}{l}2.12(-0.78 ; 5.03) \\
{[\mathrm{N}=493]} \\
(\mathrm{p}=0.152)\end{array}$ & $\begin{array}{l}4.53(0.68 ; 8.38) \\
{[N=169]} \\
(p=0.021)\end{array}$ \\
\hline Diarrhea age 15 (self) & $\begin{array}{l}4.18(1.52 ; 6.83) \\
(p=0.002)^{*}\end{array}$ & $\begin{array}{l}5.15(2.74 ; 7.55) \\
(p<0.001)^{*}\end{array}$ & $\begin{array}{l}2.67(-1.87 ; 7.19) \\
{[N=173]} \\
(p=0.250)\end{array}$ & $\begin{array}{l}0.29(-5.22 ; 5.81) \\
{[N=76]} \\
(p=0.917)\end{array}$ \\
\hline C/D age 15 (self) & $\begin{array}{l}10.84(5.47 ; 16.21) \\
(p<0.001)^{*}\end{array}$ & $\begin{array}{l}9.52(4.27 ; 14.75) \\
(p<0.001)^{*}\end{array}$ & $\begin{array}{l}5.58(-1.89 ; 13.05) \\
{[N=71]} \\
(p=0.143)\end{array}$ & $\begin{array}{l}4.50(-3.99 ; 12.99) \\
{[N=25]} \\
(p=0.299)\end{array}$ \\
\hline
\end{tabular}

*Indicates $p$-values that are below the statistical significant threashold set at $p<.005$.

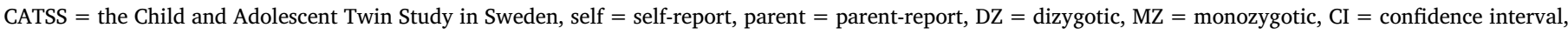
EDI $=$ Eating Disorders Inventory-2, C/D = reporting both constipation and diarrhea.

a Adjusted for sex.

b Number of individuals from exposure discordant twin pairs in analysis.

Table 3

Results from regression analyses (regression coefficients, with 95\% confidence intervals (CI) and p-values) evaluating associations between EDI total score at age 18 and constipation, diarrhea, and reporting both GI conditions (C/D) at ages 9/12 and 15 in CATSS. Crude models, models adjusted for sex, and within pair models (DZ and MZ twin) are presented.

\begin{tabular}{|c|c|c|c|c|}
\hline & $\begin{array}{l}\text { Crude } \\
(95 \% \mathrm{CI})\end{array}$ & $\begin{array}{l}\text { Adjusted }^{\mathrm{a}} \\
(95 \% \mathrm{CI})\end{array}$ & $\begin{array}{l}\text { Within DZ pairs } \\
\text { (95\% CI) } \\
{\left[\mathrm{N}=\text { exposure discordant twins }{ }^{\mathrm{b}}\right]}\end{array}$ & $\begin{array}{l}\text { Within MZ pairs } \\
(95 \% \mathrm{CI}) \\
{\left[\mathrm{N}=\text { exposure discordant twins }{ }^{\mathrm{b}}\right]}\end{array}$ \\
\hline Constipation age $9 / 12$ (parent) & $\begin{array}{l}4.10(1.83 ; 6.35) \\
(p<0.001)^{*}\end{array}$ & $\begin{array}{l}1.97(-0.09 ; 4.02) \\
(p=0.061)\end{array}$ & $\begin{array}{l}-0.31(-3.49 ; 2.88) \\
{[\mathrm{N}=473]} \\
(\mathrm{p}=0.849)\end{array}$ & $\begin{array}{l}2.07(-1.70 ; 5.84) \\
{[\mathrm{N}=135]} \\
(\mathrm{p}=0.281)\end{array}$ \\
\hline Constipation age 15 (self) & $\begin{array}{l}7.46(4.16 ; 10.76) \\
(p<0.001)^{* *}\end{array}$ & $\begin{array}{l}5.04(1.97 ; 8.10) \\
(\mathrm{p}=0.001)^{*}\end{array}$ & $\begin{array}{l}1.11(-4.54 ; 6.77) \\
{[N=185]} \\
(\mathrm{p}=0.699)\end{array}$ & $\begin{array}{l}1.69(-4.90 ; 8.28) \\
{[N=65]} \\
(p=0.615)\end{array}$ \\
\hline Diarrhea age 15 (self) & $\begin{array}{l}0.80(-3.86 ; 5.45) \\
(\mathrm{p}=0.738)\end{array}$ & $\begin{array}{l}1.43(-2.76 ; 5.63) \\
(\mathrm{p}=0.504)\end{array}$ & $\begin{array}{l}-0.55(-8.16 ; 9.27) \\
{[N=65]} \\
(p=0.901)\end{array}$ & $\begin{array}{l}-2.41(-8.99 ; 4.17) \\
{[\mathrm{N}=42]} \\
(\mathrm{p}=0.473)\end{array}$ \\
\hline C/D age 15 (self) & $\begin{array}{l}8.12(-0.94 ; 17.17) \\
(\mathrm{p}=0.079)\end{array}$ & $\begin{array}{l}5.58(-2.41 ; 13.57) \\
(\mathrm{p}=0.171)\end{array}$ & $\begin{array}{l}6.60(-8.15 ; 21.35) \\
{[N=17]} \\
(p=0.380)\end{array}$ & $\begin{array}{l}-3.83(-18.36 ; 10.69) \\
{[N=13]} \\
(p=0.605)\end{array}$ \\
\hline
\end{tabular}

"Indicates p-values that are below the statistical significant threashold set at $\mathrm{p}<.005$.

CATSS $=$ the Child and Adolescent Twin Study in Sweden, self $=$ self-report, parent $=$ parent report, $\mathrm{DZ}=$ dizygotic, $\mathrm{MZ}=$ monozygotic, $\mathrm{CI}=$ confidence interval, $\mathrm{EDI}=$ Eating Disorders Inventory-2, C/D = constipation and diarrhea.

a Adjusted for sex.

${ }^{b}$ Number of individuals from exposure discordant twin pairs in analysis.

at age 15: linear regression showed positive, significant associations among constipation, diarrhea, and C/D with all three EDI sub-scales. For the EDI sub-scales measured at age 18 , only constipation at age 15 , and not diarrhea, was significantly associated with the BD and B subscales. In the within-twin pair analysis, no estimates remained significant. Because the associations between constipation and diarrhea with the EDI total score were not significant at age $9 / 12$, we did not further examine the sub-scale scores.

\subsection{Sensitivity analysis}

We examined the association between self-reported constipation at age 15 and EDI total score at age 18, adjusting for EDI total score at age 15. The magnitude of the association decreased compared to the unadjusted results and was not significant, regression coefficients of 0.75 (95\% CI: $-1.73 ; 3.23)$. We did not proceed with a sensitivity analysis between diarrhea at age 15 and EDI total score at age 18 adjusted for EDI total score at age 15 because there was no statistically significant association in the unadjusted analysis. 
Table 4

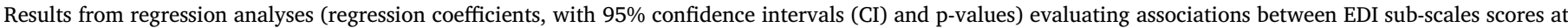

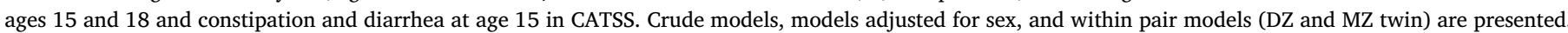

\begin{tabular}{|c|c|c|c|c|}
\hline & $\begin{array}{l}\text { Crude } \\
(95 \% \mathrm{CI})\end{array}$ & $\begin{array}{l}\text { Adjusted }^{\mathrm{a}} \\
(95 \% \mathrm{CI})\end{array}$ & $\begin{array}{l}\text { Within DZ pairs }{ }^{\mathrm{a}} \\
(95 \% \mathrm{CI}) \\
{\left[\mathrm{N}=\text { exposure discordant twins }{ }^{\mathrm{b}}\right]}\end{array}$ & $\begin{array}{l}\text { Within MZ pairs } \\
(95 \% \mathrm{CI}) \\
{\left[\mathrm{N}=\text { exposure discordant twins }{ }^{\mathrm{b}}\right]}\end{array}$ \\
\hline & \multicolumn{4}{|c|}{ Drive for thinness sub-scale age 15} \\
\hline Constipation age 15 (self) & $\begin{array}{l}2.86(2.08 ; 3.63) \\
(\mathrm{p}<0.001)^{*}\end{array}$ & $\begin{array}{l}2.11(1.39 ; 2.83) \\
(\mathrm{p}<0.001)^{*}\end{array}$ & $\begin{array}{l}1.42(0.28 ; 2.55) \\
(\mathrm{p}=0.015) \\
{[\mathrm{N}=524]}\end{array}$ & $\begin{array}{l}1.68(0.11 ; 3.25) \\
(\mathrm{p}=0.036) \\
{[\mathrm{N}=173]}\end{array}$ \\
\hline Diarrhea age 15 (self) & $\begin{array}{l}1.47(0.33 ; 2.61) \\
(p=0.012)\end{array}$ & $\begin{array}{l}1.80(0.78 ; 2.83) \\
(\mathrm{p}<0.001)^{*}\end{array}$ & $\begin{array}{l}0.76(-1.08 ; 2.59) \\
(\mathrm{p}=0.420) \\
{[\mathrm{N}=179]}\end{array}$ & $\begin{array}{l}-0.06(-2.01 ; 1.90) \\
(\mathrm{p}=0.956) \\
{[\mathrm{N}=78]}\end{array}$ \\
\hline \multirow[t]{2}{*}{ C/D age 15 (self) } & $\begin{array}{l}4.13(1.89 ; 6.36) \\
(p<0.001)^{*}\end{array}$ & $\begin{array}{l}3.62(1.44 ; 5.79) \\
(p=0.001)^{*}\end{array}$ & $\begin{array}{l}3.21(0.63 ; 5.79) \\
(\mathrm{p}=0.015) \\
{[\mathrm{N}=74]}\end{array}$ & $\begin{array}{l}0.36(-2.66 ; 3.39) \\
(\mathrm{p}=0.814) \\
{[\mathrm{N}=27]}\end{array}$ \\
\hline & \multicolumn{4}{|c|}{ Body dissatisfaction sub-scale age 15} \\
\hline Constipation age 15 (self) & $\begin{array}{l}3.17(2.27 ; 4.07) \\
(p<0.001)^{*}\end{array}$ & $\begin{array}{l}2.24(1.40 ; 3.07) \\
(p<0.001)^{*}\end{array}$ & $\begin{array}{l}0.87(-0.50 ; 2.23) \\
(p=0.213) \\
{[N=529]}\end{array}$ & $\begin{array}{l}2.32(0.41 ; 4.24) \\
(\mathrm{p}=0.018) \\
{[\mathrm{N}=180]}\end{array}$ \\
\hline Diarrhea age 15 (self) & $\begin{array}{l}1.58(0.24 ; 2.92) \\
(p=0.021)\end{array}$ & $\begin{array}{l}2.01(0.83 ; 3.18) \\
(p<0.001)^{*}\end{array}$ & $\begin{array}{l}0.47(-1.78 ; 2.72) \\
(\mathrm{p}=0.682) \\
{[\mathrm{N}=180]}\end{array}$ & $\begin{array}{l}-0.37(-3.20 ; 2.46) \\
(\mathrm{p}=0.799) \\
{[\mathrm{N}=80]}\end{array}$ \\
\hline \multirow[t]{2}{*}{ C/D age 15 (self) } & $\begin{array}{l}4.20(1.66 ; 6.75) \\
(p=0.001)^{*}\end{array}$ & $\begin{array}{l}3.44(1.07 ; 5.81) \\
(p=0.004)^{*}\end{array}$ & $\begin{array}{l}1.05(-2.75 ; 4.85) \\
(\mathrm{p}=0.587) \\
{[\mathrm{N}=73]}\end{array}$ & $\begin{array}{l}0.75(-3.95 ; 5.45) \\
(\mathrm{p}=0.754) \\
{[\mathrm{N}=28]}\end{array}$ \\
\hline & Bulimia sub-scale age 15 & & & \\
\hline Constipation age 15 (self) & $\begin{array}{l}1.41(0.96 ; 1.87) \\
(p<0.001)^{*}\end{array}$ & $\begin{array}{l}1.27(0.98 ; 1.35) \\
(\mathrm{p}<0.001)^{*}\end{array}$ & $\begin{array}{l}0.23(-0.49 ; 1.01) \\
(\mathrm{p}=0.495) \\
{[\mathrm{N}=534]}\end{array}$ & $\begin{array}{l}0.43(-0.50 ; 1.36) \\
(\mathrm{p}=0.368) \\
{[\mathrm{N}=182]}\end{array}$ \\
\hline Diarrhea age 15 (self) & $\begin{array}{l}2.05(1.37 ; 2.74) \\
(p<0.001)^{*}\end{array}$ & $\begin{array}{l}2.11(1.42 ; 2.80) \\
(p<0.001)^{*}\end{array}$ & $\begin{array}{l}1.77(0.73 ; 3.06) \\
(\mathrm{p}=0.007) \\
{[\mathrm{N}=183]}\end{array}$ & $\begin{array}{l}0.44(-1.15 ; 2.04) \\
(\mathrm{p}=0.590) \\
{[\mathrm{N}=83]}\end{array}$ \\
\hline \multirow[t]{2}{*}{ C/D age 15 (self) } & $\begin{array}{l}3.16(1.73 ; 4.59) \\
(\mathrm{p}<0.001)^{*}\end{array}$ & $\begin{array}{l}3.03(1.57 ; 4.49) \\
(p<0.001)^{*}\end{array}$ & $\begin{array}{l}2.37(-0.15 ; 4.89) \\
(p=0.065) \\
{[N=74]}\end{array}$ & $\begin{array}{l}2.31(0.24 ; 4.38) \\
(\mathrm{p}=0.029) \\
{[\mathrm{N}=29]}\end{array}$ \\
\hline & Drive for thinness sub-sco & & & \\
\hline Constipation age 15 (self) & $\begin{array}{l}2.34(1.05 ; 3.64) \\
(p<0.001)\end{array}$ & $\begin{array}{l}1.46(0.27 ; 2.65) \\
(p=0.016)\end{array}$ & $\begin{array}{l}0.03(-2.12 ; 2.17) \\
(\mathrm{p}=0.981) \\
{[\mathrm{N}=189]}\end{array}$ & $\begin{array}{l}1.11(-1.52 ; 3.75) \\
(\mathrm{p}=0.408) \\
{[\mathrm{N}=67]}\end{array}$ \\
\hline Diarrhea age 15 (self) & $\begin{array}{l}0.01(-1.92 ; 1.94) \\
(p=0.990)\end{array}$ & $\begin{array}{l}0.45(-1.25 ; 2.15) \\
(\mathrm{p}=0.605)\end{array}$ & $\begin{array}{l}0.13(-4.05 ; 4.31) \\
(\mathrm{p}=0.950) \\
{[\mathrm{N}=68]}\end{array}$ & $\begin{array}{l}-1.78(-4.83 ; 1.28) \\
(\mathrm{p}=0.254) \\
{[\mathrm{N}=43]}\end{array}$ \\
\hline \multirow[t]{2}{*}{ C/D age 15 (self) } & $\begin{array}{l}3.16(-0.56 ; 6.87) \\
(p=0.096)\end{array}$ & $\begin{array}{l}2.34(-1.07 ; 5.76) \\
(\mathrm{p}=0.179)\end{array}$ & $\begin{array}{l}7.00(0.26 ; 13.74) \\
(\mathrm{p}=0.042) \\
{[\mathrm{N}=18]}\end{array}$ & $\begin{array}{l}0.17(-7.09 ; 7.42) \\
(\mathrm{p}=0.964) \\
{[\mathrm{N}=13]}\end{array}$ \\
\hline & Body dissatisfaction sub-s & 18 & & \\
\hline Constipation age 15 (self) & $\begin{array}{l}3.12(1.62 ; 4.62) \\
(\mathrm{p}<0.001)^{*}\end{array}$ & $\begin{array}{l}2.12(0.72 ; 3.51) \\
(p=0.003)^{*}\end{array}$ & $\begin{array}{l}0.08(-2.58 ; 2.73) \\
(\mathrm{p}=0.956) \\
{[\mathrm{N}=188]}\end{array}$ & $\begin{array}{l}-0.32(-3.43 ; 2.78) \\
(\mathrm{p}=0.839) \\
{[\mathrm{N}=68]}\end{array}$ \\
\hline Diarrhea age 15 (self) & $\begin{array}{l}-0.17(-2.30 ; 1.96) \\
(p=0.874)\end{array}$ & $\begin{array}{l}0.11(-1.84 ; 2.06) \\
(p=0.913)\end{array}$ & $\begin{array}{l}-1.72(-5.79 ; 2.35) \\
(\mathrm{p}=0.408) \\
{[\mathrm{N}=69]}\end{array}$ & $\begin{array}{l}0.06(-3.32 ; 3.43) \\
(\mathrm{p}=0.974) \\
{[\mathrm{N}=43]}\end{array}$ \\
\hline C/D age 15 (self) & $\begin{array}{l}3.66(-0.63 ; 7.95) \\
(p=0.094)\end{array}$ & $\begin{array}{l}2.50(-1.26 ; 6.27) \\
(p=0.193)\end{array}$ & $\begin{array}{l}4.40(0.74 ; 8.06) \\
(\mathrm{p}=0.019) \\
{[\mathrm{N}=17]}\end{array}$ & $\begin{array}{l}-2.83(-10.72 ; 5.06) \\
(\mathrm{p}=0.482) \\
{[\mathrm{N}=13]}\end{array}$ \\
\hline Constipation age 15 (self) & $\begin{array}{l}\text { Bulimia sub-scale age } 18 \\
1.67(0.76 ; 2.58) \\
(p<0.001)^{*}\end{array}$ & $\begin{array}{l}1.37(0.47 ; 2.27) \\
(\mathrm{p}=0.003)^{*}\end{array}$ & $\begin{array}{l}0.93(-1.00 ; 2.86) \\
(\mathrm{p}=0.346) \\
{[\mathrm{N}=188]}\end{array}$ & $\begin{array}{l}0.81(-1.28 ; 2.91) \\
(\mathrm{p}=0.446) \\
{[\mathrm{N}=66]}\end{array}$ \\
\hline Diarrhea age 15 (self) & $\begin{array}{l}0.85(-0.35 ; 2.05) \\
(p=0.166)\end{array}$ & $\begin{array}{l}0.97(-0.23 ; 2.16) \\
(\mathrm{p}=0.113)\end{array}$ & $\begin{array}{l}1.47(-1.05 ; 4.00) \\
(\mathrm{p}=0.252) \\
{[\mathrm{N}=70]}\end{array}$ & $\begin{array}{l}-1.53(-3.50 ; 0.45) \\
(\mathrm{p}=0.130) \\
{[\mathrm{N}=44]}\end{array}$ \\
\hline C/D age 15 (self) & $\begin{array}{l}1.91(-0.46 ; 4.28) \\
(p=0.113)\end{array}$ & $\begin{array}{l}1.67(-0.74 ; 4.08) \\
(p=0.174)\end{array}$ & $\begin{array}{l}-1.33(-7.43 ; 4.77) \\
(\mathrm{p}=0.668) \\
{[\mathrm{N}=18]}\end{array}$ & $\begin{array}{l}-1.17(-4.38 ; 2.05) \\
(\mathrm{p}=0.477) \\
{[\mathrm{N}=13]}\end{array}$ \\
\hline
\end{tabular}

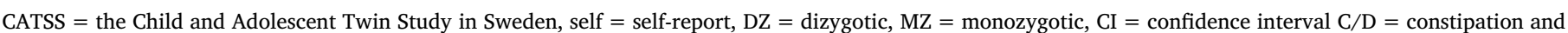
diarrhea.

a Adjusted for sex.

b Number of individuals from exposure discordant twin pairs in analysis.

* Indicates p-values that are below the statistical significant threashold set at $\mathrm{p}<0.005$. 


\section{Discussion}

The results from this study show a positive association between GI problems in childhood and early adolescence, and later disordered eating. Individuals who reported a history of diarrhea at age 15 scored approximately 5 points higher on the EDI scale reported at age 15 than those who reported no GI problems. Individuals who reported a history of constipation at age 15 scored approximately 5 points higher on the EDI scale reported at age 15 and at age 18. Individuals who reported both conditions at age 15 scored over 9 points higher than those reporting no GI problems. Further, we observed that the association between constipation and disordered eating decreased when we conditioned the analysis within the twin pairs; however, it remained positive. This could be interpreted as constipation having a partially direct effect on disordered eating, or vice versa. The association observed between constipation at age 15 and disordered eating at age 18 was no longer significant when controlling for disordered eating at age 15 . This attenuation could be explained by one or both of two explanations: 1) disordered eating at age 15 is a confounding factor affecting both the exposure (constipation at age 15) and the outcome (disordered eating at age 18), and/or 2) disordered eating at age 15 is a mediating factor on the path between constipation at age 15 and disordered eating at age 18. Unfortunately, we cannot further disentangle this relationship due to the fact that constipation and disordered eating are both recorded at the same timepoint (age 15).

In contrast to the association between constipation and EDI, we do not see a significant association between diarrhea and EDI over the same time period. The standardized effect size for self-reported constipation is stable across the two ages ( 0.42 and 0.39 respectively), in contrast the standardized effect size for self-reported diarrhea is smaller at age 15 and declines further at age 18 ( 0.26 and 0.12 respectively). However, even if the sample were large enough to detect the smaller effect size at age 18, this potential association would most likely not be of clinical importance.

Two previous studies have reported GI problems in childhood to be associated with the development of eating disorders. Råstam et al. reported that childhood GI problems (defined as early feeding problems, severe bellyache, or treatment in hospital for GI problems) were significantly more common in their sample of 51 adolescent females with anorexia nervosa than in healthy controls [28]. In an Israeli cross-sectional study of a population sample of adolescent school girls, constipation was associated with the later development of disordered eating [29]. Our study extends this work by suggesting that this observed relationship is partly a direct effect of the exposure (GI problems) on the outcome (disordered eating) and partly due to familial confounding.

The familial confounding could be due to different types of geneenvironment correlations that occur when genetic predispositions are expressed differently in different environments [30]. For example, constipation leads to slow colonic transit time, which can contribute to bloating [4]. Bloating could contribute to body dissatisfaction and could be misperceived by individuals predisposed to eating disorders as being larger than desired. A perceived discrepancy between one's selfperception and the societal thin ideal, even if the discrepancy is due to frequent bloating, could increase the risk of disordered eating in order to control shape or weight. This could account for why we observe an association between constipation at age 15 and the EDI total score, but not with constipation at age $9 / 12$, at an age when the impact of the societal thin ideal is less pervasive [31].

With reference to the potential causal/direct association, GI problems in childhood could also lead to increased somatic awareness of the GI tract thereby increasing visceral sensitivity, which is associated with the intensity of GI symptoms [32]. A heightened focus on GI symptoms might also lead to increased concern and awareness about diet which, in turn, could predispose to disordered eating behaviors. A majority of patients with IBS report their GI symptoms to be triggered by certain food items (especially carbohydrates and fat). Additionally, reporting a high number of food items causing GI symptoms was associated with reduced quality of life [33]. One review found that symptom severity and dietary adherence was associated with disordered eating patterns in individuals with GI disorders [34]. The authors suggested that low adherence to a dietary routine (e.g., continued consumption of trigger food) could be a way of promoting weight loss. In contrast, the dietary management prescribed for many GI problems could lead some individuals to become highly anxious and preoccupied with food choices to control GI symptoms thereby playing a causal role in the development of disordered eating patterns [34].

Another possible direct association includes the observation that prolonged diarrhea is common in children in pediatric clinics and can be a sign of multiple problems, including lactose intolerance and celiac disease [35]. Likewise, food allergies and intolerances have been found to be associated with eating disorders and other psychiatric symptoms such as anxiety and depression [36,37], which are known to be common in children with pediatric recurrent abdominal pain [14,38].

Although the findings from our study suggest that there are possible direct or even causal mechanisms linking GI problems with disordered eating, it will be important to expand and replicate current findings in clinically diagnosed samples of individuals with eating disorders. In addition, the results of this study could be influenced by unknown environmental risk factors affecting both GI problems and disordered eating leading to a causal interpretation of the relationship. One such potential risk factor could be involvement of the bacterial community situated in the intestines (i.e., the intestinal microbiota). Associations have been reported between a dysbiosis (imbalance) in the intestinal microbiota and several key features of both eating disorders and GI problems, such as weight regulation and energy metabolism [39], mood and behavior [40,41], constipation in children [42,43], inflammatory bowel disease in adults [44], and eating disorders [45]. Direct and longitudinal sampling is required to evaluate whether the intestinal microbiota plays a uniting causal role in GI problems and eating disorders.

Several limitations to this study should be considered. First, we accessed data that were a combination of parent- and self-report interviews and questionnaires. Second, our sample is large and population-based and we did not have access to clinical diagnoses of either GI problems or eating disorders. In addition, the questions regarding GI problems have not been formally validated, meaning we cannot ensure that the questions are measuring what they are supposed to measure. Third, we used three EDI sub-scales as an index of disordered eating. In validation studies, the EDI has been reported to discriminate well between eating disorder patient groups and healthy controls [21,23]. The focus of this study was not to detect eating disorders, but rather to explore whether risk of disordered eating was elevated in a general population sample in individuals exposed to prolonged diarrhea or constipation. Fourth, we focused on constipation and diarrhea as they were the only measures of GI symptoms available in the cohort. We acknowledge that abdominal pain would have been of particular interest given its association with GI disorders in adulthood [10,11]; however, it was not assessed in CATSS. To further clarify our findings and their relevance to threshold eating disorders, longitudinal clinical and register studies are needed. Fifth, GI problems were retrospectively reported and cover the whole childhood period until the time of assessment. We are therefore unable to disentangle childhood and adolescent GI problems. However, the two time points of evaluating GI problems in this study (age 9/12 and age 15) suggest that the association we observe reflects adolescent rather than early childhood problems or the accuracy of self- versus parent-report. It is possible that the self-reported measures at age 15 reflect GI problems closer to the time of assessment, compared to the parent-reported measures of symptoms at age $9 / 12$.

Sixth, our results do not rule out reverse causality, nor can we draw conclusions regarding temporality. We cannot determine from our data 
whether the GI problems occurred before or after the disordered eating emerged. As previously stated, GI problems are common in patients with eating disorders; however, this is not a clinical sample and eating disorders (especially binge-eating disorder and bulimia nervosa rather than anorexia nervosa) typically onset in later adolescence or early adulthood $[46,47]$. Nevertheless, we cannot rule out that early onset of disordered eating, for example reduced food intake, and a selective and poor diet could lead to GI problems such as constipation.

Seventh, this study is also influenced by the limitations that are inherent in using a within-twin pair design such as potential unmeasured confounding factors that are causing the differences in the exposure and that also may be responsible for the differences in the outcome [25].

Finally, we view this investigation as preliminary given the limited available assessments of childhood GI problems. We encourage future investigations with more extensive phenotyping to further explore the relation between childhood GI problems and disordered eating and eating disorders in later adolescence.

In conclusion, our findings show an association between childhood GI problems and disordered eating, which is, in part, due to genetic and/or familial confounding. Clinicians should be alert for disordered eating when treating children and adolescents presenting with prolonged constipation or diarrhea and potentially other GI problems. Future studies should focus on exploring specific mechanisms that may influence both the development of GI problems and disordered eating using both clinical and molecular genetic designs.

\section{Funding}

This work was supported by the Swedish Research Council (VR Dnr: 538-2013-8864).

\section{Declaration of Competing Interest}

Cynthia Bulik is a grant recipient from and has served on advisory boards for Shire and received royalties from Pearson. The other authors have no competing interests to report.

\section{Acknowledgements}

We acknowledge The Swedish Twin Registry for access to data. The Swedish Twin Registry is managed by Karolinska Institutet and receives funding through the Swedish Research Council (VR Dnr: 2017-00641). Dr. Bulik acknowledges funding from the Swedish Research Council (VR Dnr: 538-2013-8864).

\section{References}

[1] F. Cremonini, M. Camilleri, M.M. Clark, T.J. Beebe, G.R. Locke, A.R. Zinsmeister, et al., Associations among binge eating behavior patterns and gastrointestinal symptoms: a population-based study, Int. J. Obes. 33 (3) (2009) 342-353, https:// doi.org/10.1038/ijo.2008.272.

[2] S. Zipfel, I. Sammet, N. Rapps, W. Herzog, S. Herpertz, U. Martens, Gastrointestinal disturbances in eating disorders: clinical and neurobiological aspects, Auton. Neurosci. 129 (1-2) (2006) 99-106, https://doi.org/10.1016/j.autneu.2006.07. 023.

[3] C.M. Peat, L. Huang, L.M. Thornton, A.F. Von Holle, S.E. Trace, P. Lichtenstein, et al., Binge eating, body mass index, and gastrointestinal symptoms, J. Psychosom. Res. 75 (5) (2013) 456-461, https://doi.org/10.1016/j.jpsychores.2013.08.009.

[4] Y. Sato, S. Fukudo, Gastrointestinal symptoms and disorders in patients with eating disorders, Clin. J. Gastroenterol. 8 (5) (2015) 255-263, https://doi.org/10.1007/ s12328-015-0611-x.

[5] E.M. Bern, R.F. O'Brien, Is it an eating disorder, gastrointestinal disorder, or both? Curr. Opin. Pediatr. 25 (4) (2013) 463-470, https://doi.org/10.1097/MOP. ob013e328362d1ad.

[6] C. Boyd, S. Abraham, J. Kellow, Psychological features are important predictors of functional gastrointestinal disorders in patients with eating disorders, Scand. J. Gastroenterol. 40 (8) (2005) 929-935, https://doi.org/10.1080/ 00365520510015836.

[7] G. Stacher, Gut function in anorexia nervosa and bulimia nervosa, Scand. J. Gastroenterol. 38 (6) (2003) 573-587, https://doi.org/10.1080/
00365520310002120

[8] Y. Vandenplas, A. Abkari, M. Bellaiche, M. Benninga, J.P. Chouraqui, F. Cokura, et al., Prevalence and health outcomes of functional gastrointestinal symptoms in infants from birth to 12 months of age, J. Pediatr. Gastroenterol. Nutr. 61 (5) (2015) 531-537, https://doi.org/10.1097/mpg.0000000000000949.

[9] D.K. Chitkara, D.J. Rawat, N.J. Talley, The epidemiology of childhood recurrent abdominal pain in Western countries: a systematic review, Am. J. Gastroenterol. 100 (8) (2005) 1868-1875, https://doi.org/10.1111/j.1572-0241.2005.41893.x.

[10] D.K. Chitkara, N.J. Talley, C. Schleck, A.R. Zinsmeister, N.D. Shah, G.R. Locke 3rd, Recollection of childhood abdominal pain in adults with functional gastrointestinal disorders, Scand. J. Gastroenterol. 44 (3) (2009) 301-307, https://doi.org/10. 1080/00365520802555975.

[11] S. Horst, G. Shelby, J. Anderson, S. Acra, D.B. Polk, B.R. Saville, et al., Predicting persistence of functional abdominal pain from childhood into young adulthood, Clin. Gastroenterol. Hepatol. 12 (12) (2014) 2026-2032, https://doi.org/10.1016/ j.cgh.2014.03.034.

[12] J.V. Campo, C. Di Lorenzo, L. Chiappetta, J. Bridge, D.K. Colborn, J.C. Gartner Jr.et al., Adult outcomes of pediatric recurrent abdominal pain: do they just grow out of it? Pediatrics. 108 (1) (2001) E1, https://doi.org/10.1542/peds.108.1.e1.

[13] J.J. Korterink, K. Diederen, M.A. Benninga, M.M. Tabbers, Epidemiology of pediatric functional abdominal pain disorders: a meta-analysis, PLoS One 10 (5) (2015) e0126982, , https://doi.org/10.1371/journal.pone.0126982.

[14] J.V. Campo, J. Bridge, M. Ehmann, S. Altman, A. Lucas, B. Birmaher, et al., Recurrent abdominal pain, anxiety, and depression in primary care, Pediatrics. 113 (4) (2004) 817-824, https://doi.org/10.1111/j.1365-2214.2004.00456_1.x.

[15] J. Taljemark, M. Rastam, P. Lichtenstein, H. Anckarsater, N. Kerekes, The coexistence of psychiatric and gastrointestinal problems in children with restrictive eating in a nationwide Swedish twin study, J. Eat. Disord. 5 (2017) 25, https://doi. org/10.1186/s40337-017-0154-2.

[16] S. Zerwas, J.T. Larsen, L. Petersen, L.M. Thornton, M. Quaranta, S.V. Koch, et al., Eating disorders, autoimmune, and autoinflammatory disease, Pediatrics. 140 (6) (2017) e20162089, , https://doi.org/10.1542/peds.2016-2089.

[17] D.M. Garner, Eating Disorder Inventory-2: Professional Manual, Psycologica Assessment Resourses Inc, Odessa, FL, 1991.

[18] H. Anckarsäter, S. Lundström, L. Kollberg, N. Kerekes, C. Palm, E. Carlström, et al., The child and adolescent twin study in Sweden (CATSS), Twin Res. Hum. Genet. 14 (6) (2012) 495-508, https://doi.org/10.1375/twin.14.6.495.

[19] P.K. Magnusson, C. Almqvist, I. Rahman, A. Ganna, A. Viktorin, H. Walum, et al., The Swedish twin registry: establishment of a biobank and other recent developments, Twin Res. Hum. Genet. 16 (1) (2013) 317-329, https://doi.org/10.1017/ thg.2012.104.

[20] P. Lichtenstein, U. De Faire, B. Floderus, M. Svartengren, P. Svedberg, N.L. Pedersen, The Swedish twin registry: a unique resource for clinical, epidemiological and genetic studies, J. Intern. Med. 252 (3) (2002) 184-205, https://doi. org/10.1046/j.1365-2796.2002.01032.x.

[21] L. Clausen, K. Rokkedal, J.H. Rosenvinge, Validating the eating disorder inventory (EDI-2) in two Danish samples: a comparison between female eating disorder patients and females from the general population, Eur. Eat. Disord. Rev. 17 (6) (2009) 462-467, https://doi.org/10.1002/erv.945.

[22] L. Nevonen, A.G. Broberg, Validating the eating disorder Inventory-2 (EDI-2) in Sweden, Eating Weight Disord. 6 (2) (2001) 59-67, https://doi.org/10.1007/ BF03339754.

[23] L. Nevonen, D. Clinton, C. Norring, Validating the EDI-2 in three Swedish female samples: eating disorders patients, psychiatric outpatients and normal controls, Nord J. Psychiatry. 60 (1) (2006) 44-50, https://doi.org/10.1080/ 08039480500504537.

[24] J. Zetterqvist, S. Vansteelandt, Y. Pawitan, A. Sjolander, Doubly robust methods for handling confounding by cluster, Biostatistic. 17 (2) (2016) 264-276, https://doi. org/10.1093/biostatistics/kxv041.

[25] M. McGue, M. Osler, K. Christensen, Causal inference and observational research: the utility of twins, Perspect. Psychol. Sci. 5 (5) (2010) 546-556, https://doi.org/ $10.1177 / 1745691610383511$.

[26] R Core Team, R: A Language and Environment for Statistical Computing, R Foundation for Statistical Computing, Vienna, Austria, 2017.

[27] J. Zetterqvist, A. Sjölander, Doubly robust estimation with the R package drgee, Epidemiol. Methods 4 (1) (2015) 69-86, https://doi.org/10.1515/em-2014-0021.

[28] M. Råstam, Anorexia nervosa in 51 Swedish adolescents: premorbid problems and comorbidity, J. Am. Acad. Child Adolesc. Psychiatry 31 (5) (1992) 819-829, https://doi.org/10.1097/00004583-199209000-00007.

[29] D.N. Kaluski, B.K. Natamba, R. Goldsmith, T. Shimony, E.M. Berry, Determinants of disordered eating behaviors among Israeli adolescent girls, Eat. Disord. 16 (2) (2008) 146-159, https://doi.org/10.1080/10640260801887303.

[30] M.T. Tsuang, J.L. Bar, W.S. Stone, S.V. Faraone, Gene-environment interactions in mental disorders, World Psychiatry 3 (2) (2004) 73-83.

[31] J.L. Suisman, J.K. Thompson, P.K. Keel, S.A. Burt, M. Neale, S. Boker, et al., Genetic and environmental influences on thin-ideal internalization across puberty and preadolescent, adolescent, and young adult development, Int. J. Eat Disord. 47 (7) (2014) 773-783, https://doi.org/10.1002/eat.22321.

[32] M. Simren, H. Tornblom, O.S. Palsson, M.A.L. van Tilburg, L. Van Oudenhove, J. Tack, et al., Visceral hypersensitivity is associated with GI symptom severity in functional GI disorders: consistent findings from five different patient cohorts, Gut 67 (2) (2018) 255-262, https://doi.org/10.1136/gutjnl-2016-312361.

[33] L. Bohn, S. Storsrud, H. Tornblom, U. Bengtsson, M. Simren, Self-reported foodrelated gastrointestinal symptoms in IBS are common and associated with more severe symptoms and reduced quality of life, Am. J. Gastroenterol. 108 (5) (2013) 634-641, https://doi.org/10.1038/ajg.2013.105. 
[34] R. Satherley, R. Howard, S. Higgs, Disordered eating practices in gastrointestinal disorders, Appetite. 84 (2015) 240-250, https://doi.org/10.1016/j.appet.2014.10. 006.

[35] A. Guarino, A. Lo Vecchio, R. Berni Canani, Chronic diarrhoea in children, Best Pract. Res. Clin. Gastroenterol. 26 (5) (2012) 649-661, https://doi.org/10.1016/j. bpg.2012.11.004.

[36] L. Shanahan, N. Zucker, W.E. Copeland, E.J. Costello, A. Angold, Are children and adolescents with food allergies at increased risk for psychopathology? J. Psychosom. Res. 77 (6) (2014) 468-473, https://doi.org/10.1016/j.jpsychores. 2014.10.005.

[37] M. Teufel, T. Biedermann, N. Rapps, C. Hausteiner, P. Henningsen, P. Enck, et al., Psychological burden of food allergy, World J. Gastroenterol. 13 (25) (2007) 3456-3465, https://doi.org/10.3748/wjg.v13.i25.3456.

[38] G.D. Shelby, K.C. Shirkey, A.L. Sherman, J.E. Beck, K. Haman, A.R. Shears, et al., Functional abdominal pain in childhood and long-term vulnerability to anxiety disorders, Pediatrics. 132 (3) (2013) 475-482, https://doi.org/10.1542/peds.20122191.

[39] V.K. Ridaura, J.J. Faith, F.E. Rey, J. Cheng, A.E. Duncan, A.L. Kau, et al., Gut microbiota from twins discordant for obesity modulate metabolism in mice, Science. 341 (6150) (2013) 1241214, , https://doi.org/10.1126/science.1241214.

[40] N. Sudo, Y. Chida, Y. Aiba, J. Sonoda, N. Oyama, X.N. Yu, et al., Postnatal microbial colonization programs the hypothalamic-pituitary-adrenal system for stress response in mice, J. Physiol. 558 (Pt 1) (2004) 263-275, https://doi.org/10.1113/ jphysiol.2004.063388.

[41] R. Diaz Heijtz, S. Wang, F. Anuar, Y. Qian, B. Bjorkholm, A. Samuelsson, et al.,
Normal gut microbiota modulates brain development and behavior, Proc. Natl. Acad. Sci. U. S. A. 108 (7) (2011) 3047-3052, https://doi.org/10.1073/pnas. 1010529108.

[42] L. Zhu, W. Liu, R. Alkhouri, R.D. Baker, J.E. Bard, E.M. Quigley, et al., Structural changes in the gut microbiome of constipated patients, Physiol. Genomics 46 (18) (2014) 679-686, https://doi.org/10.1152/physiolgenomics.00082.2014.

[43] T.G. de Meij, E.F. de Groot, A. Eck, A.E. Budding, C.M. Kneepkens, M.A. Benninga, et al., Characterization of microbiota in children with chronic functional constipation, PLoS One 11 (10) (2016) e0164731, , https://doi.org/10.1371/journal. pone.0164731.

[44] A.K. DeGruttola, D. Low, A. Mizoguchi, E. Mizoguchi, Current understanding of dysbiosis in disease in human and animal models, Inflamm. Bowel Dis. 22 (5) (2016) 1137-1150, https://doi.org/10.1097/mib.0000000000000750.

[45] S.C. Kleiman, H.J. Watson, E.C. Bulik-Sullivan, E.Y. Huh, L.M. Tarantino, C.M. Bulik, et al., The intestinal microbiota in acute anorexia nervosa and during renourishment: relationship to depression, anxiety, and eating disorder psychopathology, Psychosom. Med. 77 (9) (2015) 969-981, https://doi.org/10.1097/PSY. 0000000000000247.

[46] S. Zipfel, K.E. Giel, C.M. Bulik, P. Hay, U. Schmidt, Anorexia nervosa: aetiology, assessment, and treatment, Lancet Psychiatry 2 (12) (2015) 1099-1111, https:// doi.org/10.1016/s2215-0366(15)00356-9.

[47] F.R. Smink, D. van Hoeken, H.W. Hoek, Epidemiology of eating disorders: incidence, prevalence and mortality rates, Curr. Psychiatry Rep. 14 (4) (2012) 406-414, https://doi.org/10.1007/s11920-012-0282-y. 